\title{
J.F. Kennedy: l'Addison del Presidente
}

\section{Luciano Sterpellone}

\begin{abstract}
J.F. Kennedy Addison's disease
Astract. The Addison's disease was diagnosed to the 35th president of the United States J.F. Kennedy since the early '40s, but it was kept secret for diplomatic and political reasons. The president's Addison's disease, which most likely began during the Pacific war and was treated with atabrine, got worse in October 1947, when lethal outcome was feared. Once the president was killed, the autopsy revealed that JFK was affected by type-2 polyendocrine syndrome, an autoimmune disease characterized by the contemporary presence of Addison's disease, autoimmune thyreopathy and/or insulin-dependent diabetes mellitus. Due to the difficulties in making the diagnosis and the association with other comorbidities (including depression) JFK underwent a multi-therapeutic approach until the point he had to take 10-12 different drugs a day, among which steroids, NSADs, antibiotics, sleeping pills and supplements for anemia.
\end{abstract}

Key words: J.F. Kennedy, Addison's disease, Autopsy, Type 2 autoimmune polyendocrine syndrome

Conflict of interest: None

Ricevuto: 11 Dicembre 2012; Accettato: 18 Gennaio 2013

Si era tacitamente d'accordo nel non propalare troppe notizie sulla reale salute del candidato Kennedy alla Presidenza degli Stati Uniti, segnatamente sul morbo di Addison che lo affliggeva da anni. L'immagine di un futuro Presidente dalle traballanti condizioni di salute non avrebbe certo giovato alla sua elezione: il popolo, si sa, vuole Capi scattanti, dalla salute di ferro e perfettamente efficienti sotto ogni profilo. Tutte qualità che, a prima vista, a soli 43 anni e destinato a essere il più giovane Presidente USA, John Fitzgerald sembrava possedere. Per questo, le insistenti voci che da parte repubblicana circolavano venivano recisamente smentite dai medici del suo clan. Quel che più preoccupava gli organizzatori dei fantasmagorici show pre-elettorali era in particolare la malattia (descritta da Thomas Addison sin dal 1855 (1), ma della cui vera natura si

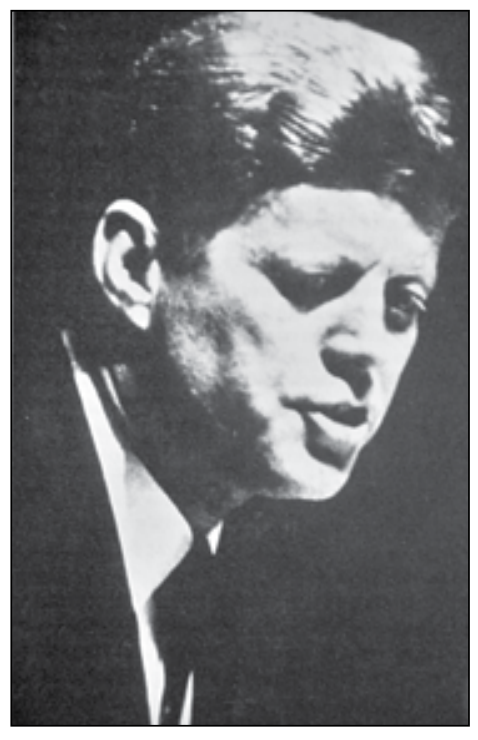

Fig. 1 sapeva ancora ben poco negli anni '40-'50) con tutti i suoi più evidenti aspetti clinici (nella sua vita Kennedy verrà ospedalizzato più di trentacinque volte, ricevendo in tre occasioni l'estrema unzione).

Un tale riserbo sarebbe persistito anche dopo la sua elezione. Anzi, "il segreto" della cartella clinica presidenziale verrà in gran parte svelato solo nel Novembre 2002, quarant'anni dopo il tragico attentato di Dallas (22 Novembre 1963): dai documenti emerge chiaramente il ruolo svolto dagli steroidi somministrati registrati nelle varie patologie del Presidente e quello che hanno svolto nella genesi e nelle complicazioni dell'Addison. Questo è stato, peraltro, sospettato sin dal 1940 e confermato sette anni dopo in coincidenza con una caduta durante un Congresso a Londra: proprio in quell'occasione gli fu prognosticato un solo anno di sopravvivenza. Una seconda caduta si verificò a Charlestown al termine di una parata di chiusura della campagna elettorale.

\section{Arriva l'Addison}

Già nell'Ottobre di quel 1947 la situazione si era improvvisamente aggravata; infatti, al futuro Presidente erano stati somministrati i sacramenti. Ma, fortunatamente, le prospettive quoad vitam migliorarono ben presto con l'avvento in terapia del cortisone e degli altri steroidi. Si ricorda, in merito, che l'Addison, codificato nel 1855, era stato considerato letale sino agli anni '30: in realtà, prima di quell'epoca, il $90 \%$ dei pazienti moriva nel giro di cinque anni. La mortalità scese precipitosamente con il progresso delle conoscenze sulla fisiologia della corteccia surrenale, con l'isolamento dei suoi ormoni, con l'avvento dei prodotti di sintesi e con la loro introduzione in terapia.

Nei decenni precedenti, essendo noto poco o nulla circa l'attività secretoria dei surreni, la malattia era stata ascritta alle cause più disparate, come la tubercolosi e la malaria. In re- 


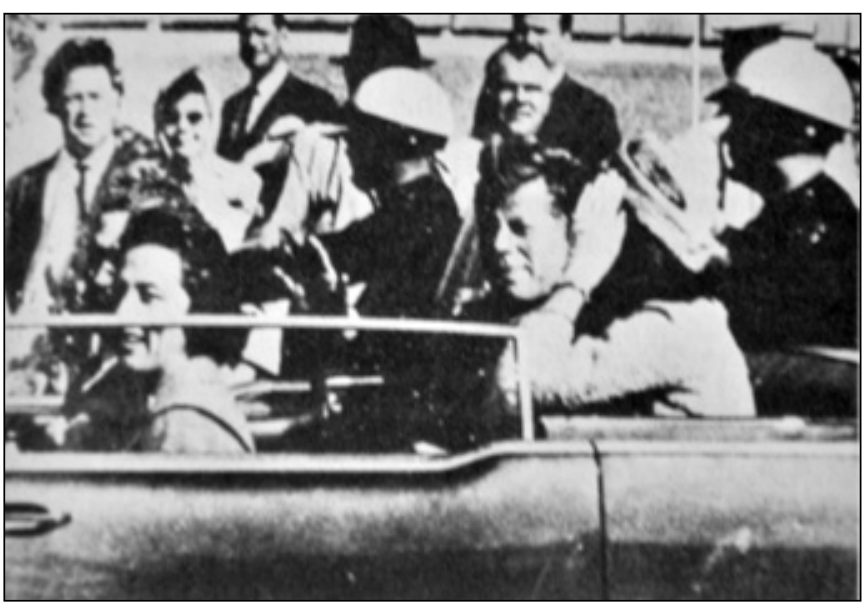

Fig. 2 - Pochi istanti prima dell'attentato.

altà, mentre non si hanno notizie di un evento tubercolare, la malaria Kennedy l'aveva contratta durante la guerra nel $\mathrm{Pa}-$ cifico e trattata con atabrina. A posteriori, è stata anche presa in considerazione la componente di una familiarità genetica tipica delle malattie autoimmuni, tenendo anche presente che la sorella più giovane di John, Eunice, era anche lei affetta dal morbo di Addison e che il figlio John Junior soffriva di un'altra malattia autoimmune, il morbo di Graves. Un'altra sorella, Rosemary, oligofrenica sin dalla nascita, a 21 anni divenne violenta e fu sottoposta "con risultati disastrosi" alla famigerata "lobotomia prefrontale" (che aveva fruttato al portoghese Caetano Moniz il Premio Nobel nel 1949) e passò il resto della vita in un'istituzione.

Per Kennedy, l'insufficienza surrenale troverà conferma nel verbale di autopsia (1963), come riferisce Lee R. Mandel, alto ufficiale medico della USA Navy, eminente endocrinologo a Chesapeake, Virginia, e tra i primi storici della medicina presidenziale a studiare a posteriori i documenti ufficiali dai quali si evinceva che, nel 1955, era stata accertata anche una condizione di ipotiroidismo.

Dall'accurato esame di quei documenti Mandel giunse alla conclusione che Kennedy era affetto dalla sindrome poliendocrina autoimmune di tipo 2 (APS-2 o sindrome di Schmidt), che comporta più patologie come "morbo di Addison o sindrome di Schmidt, ipotiroidismo e altre malattie autoimmuni".

L'Autoimmune Polyendocrine Syndrome (APS) varietà 2 (ne sono descritte $4(2)$ ) viene attualmente intesa come malattia autoimmune contrassegnata dalla presenza (costante) di morbo di Addison, associata a tireopatia autoimmune e/o a diabete mellito di tipo 1 .

Tra le potenziali cause di morbo di Addison primario sono state riconosciute, oltre alle forme autoimmuni, anche l'infezione TBC, altre infezioni, forme tumorali primitive o metastatiche del surrene, alterazioni genetiche, cause vascolari e cause chirurgiche, mentre la forma secondaria di iposurrenalismo è dovuta a un deficit di secrezione di ACTH da parte dell'ipofisi, come conseguenza di patologie infiltrative, infettive, tumorali o vascolari o di un deficit di CRH.

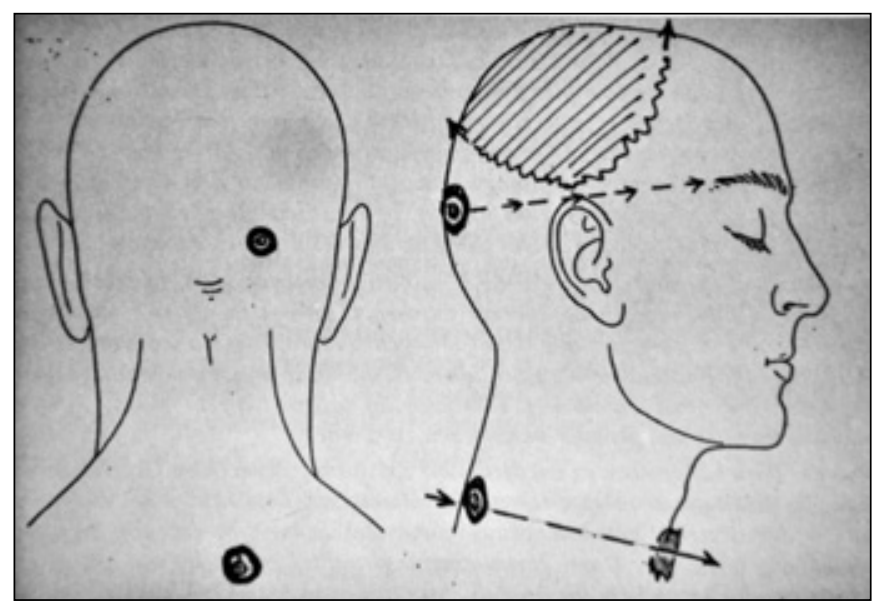

Fig. 3 - Fori di entrata e di uscita dei proiettili.

\section{Un po' di anamnesi}

Con l'utilizzo della terapia con steroidi, Kennedy diveniva sempre più paffuto ("faccia a luna piena"), il che lo faceva sembrare ancora più corpulento, ma un'altra caratteristica era quella di presentare anche un'“abbronzatura perenne", a cui sono state date varie interpretazioni.

Ha affermato Lee R. Mandel: “ $A 43$ anni JFK resta il Presidente più giovane eletto: ma, nonostante il suo apparente vigore, ha avuto la storia clinica più complessa di chiunque sia asceso alla Casa Bianca".

Per rendersi conto dell'intera patografia del Presidente e, in particolare, delle sue connessioni con il morbo di Addison, vale la pena ricordare nei suoi tratti essenziali il quadro anamnestico antecedente la sua tragica fine.

Nel 1920 (a tre anni), dopo, forse, aver sofferto di morbillo e tosse convulsa, una grave forma di scarlattina lo porta quasi in fin di vita. Per tutta l'infanzia e l'adolescenza si mostrerà sempre delicato e di salute cagionevole, soffrendo via via di difterite, asma (dopo i dieci anni), influenza, disturbi gastrici, colon irritabile e così via. Ironicamente sua madre diceva che una puntura di zanzara avrebbe potuto ucciderlo. Nel 1930 (a 13 anni) le scriverà di soffrire di obnubilamento della vista e di cecità ai colori nell'occhio destro, nonché di ipoacusia a sinistra e, a suo padre, di vertigini e svenimenti. Numerosi saranno i traumatismi subiti durante le attività sportive. Ai test scolastici rivela un IQ di 119 .

In data 21 Marzo 1931, il Dr. John Wheeler prescrive a John (quattordicenne) delle lenti per correggere una presbiopia, probabile retaggio di una difterite sofferta da bambino, come prova la positività del test di Schick effettuato nel 1926.

Il 31 Agosto del 1933 John viene sottoposto al St. Margareth Hospital ad asportazione di tonsille e adenoidi; due anni dopo sviluppa un ittero (recidivato dopo due anni) e al tempo interpretato come "iperpigmentazione addisoniana". Verrà anche colpito dalla polmonite.

Durante gli anni del College, il giovane John è alto 1.90 e pesa $67.5 \mathrm{~kg}$. Per cercare di acquistare peso fa molta palestra e con- 


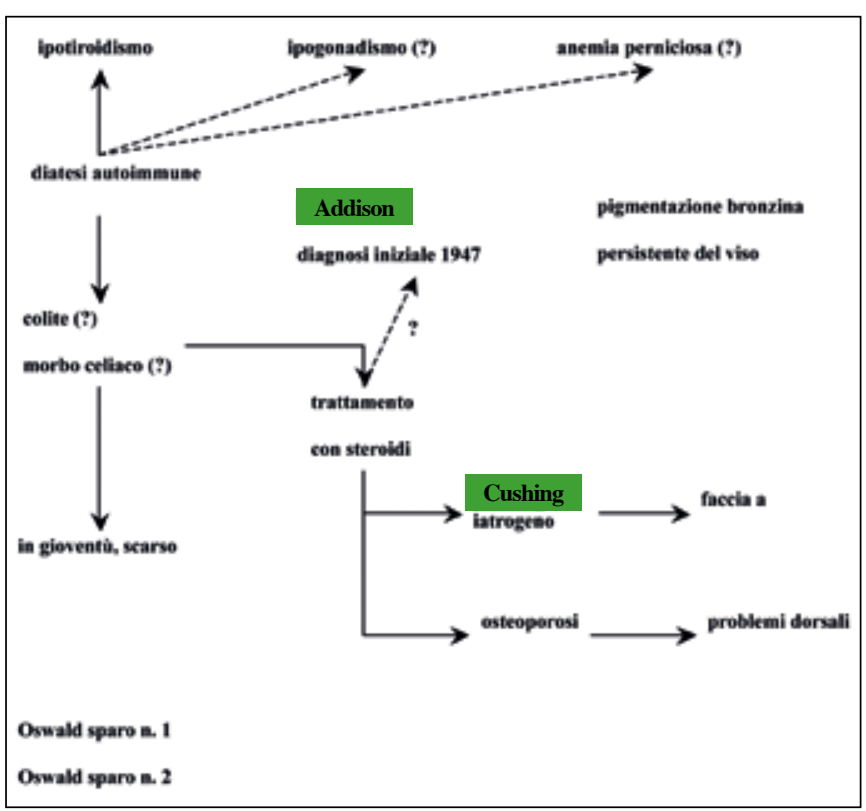

Fig. 4 - Possibili interazioni tra le patologie di JFK (3).

suma quantità "massive" di gelato. Dall'età di 20 anni e sino ai 40 il suo peso si mantiene più o meno stabile (tra i 69 e i $72 \mathrm{~kg}$ ), ma dal Luglio del 1960 al Gennaio del 1961 aumenta di 7-9 kg (annoterà la madre: "John appare insolitamente florido, con le guance piene: ha perduto il suo aspetto Lincolnesco).

Quanto al gruppo sanguigno, un documento stilato al Parkland Memorial Hospital di Dallas in occasione dell'attentato parla di sacche contenenti sangue B positivo a lui destinato, ma ne esiste anche un secondo in cui JFK era $\mathrm{AB}$ positivo. In assenza di altra documentazione è difficile dare un giudizio definitivo, anche considerando che un $\mathrm{AB}+$ può tranquillamente ricevere sangue B positivo.

Secondo lo storico Robert Dallek (della Boston University), nel 1937, a diciassette anni (1934), Kennedy soffre di disturbi intestinali, che l'anno dopo, quando entra in Marina, vengono etichettati come "colite spastica grave"; successivamente, vengono trattati con steroidi, forse in iperdosaggio con i conseguenti sintomi di ulcera duodenale, di Cushing e, per l'appunto, alla loro sospensione, di insufficienza surrenale.

Nel 1954 Kennedy viene sottoposto a un duplice intervento (assai rischioso per un addisoniano) di fusione lombo-sacrale e sacroiliaca, seguito da una massiva somministrazione di cortisone, desossicorticosterone e soluzioni saline. Il decorso postoperatorio è contrassegnato da un'infezione delle vie urinarie e da una reazione post-trasfusionale con "lieve edema angioneurotico". Verrà praticato un nuovo intervento quattro mesi dopo. Il diagramma nella Figura 4 riassume il possibile intergioco tra patologie di Kennedy e la sindrome di Addison.

Nel Maggio del 1955 la Dr.ssa Janet Travell trova un Kennedy (al tempo Senatore) "estremamente anemico che soffre di parestesie, segno di neurite periferica, tipica della carenza di vitamina B1", e prescrive iniezioni di vitamina B1, B12 e di complesso B, prontamente seguite da un aumento degli eritroci- ti e dell'emoglobina. A posteriori si è ipotizzato che si trattasse proprio di anemia perniciosa, potenziale parte della sindrome di APS (infatti l'anemia perniciosa può essere presente sia nella APS-1 che nella APS-2). Nella stessa occasione, la Travell accerta l'esistenza di un lieve ipotiroidismo (metabolismo basale $-15)$ e inizia un trattamento con iodio $(25 \mathrm{mcg} / \mathrm{die})$.

Il fatto che, durante la Presidenza, Kennedy prendesse quotidianamente pillole di testosterone ha dato adito a non poche congetture: per esempio che il suo tasso dell'ormone fosse reso deficitario dai corticosteroidi che gli venivano somministrati, oppure quale effetto dell'APS-2 (descritta come parte della sindrome (4)). In realtà, mentre, nelle femmine con morbo di Addison, è frequente un ipogonadismo ipergonadotropico che si manifesta con la menopausa precoce, nei maschi con tale patologia è dubbia l'esistenza di un ipogonadismo ipergonadotropico. Secondo altri, Kennedy assumeva testosterone per aumentare di peso: non è un caso che questo fosse aumentato notevolmente tra il 1960 e il 1961.

Certo è che il testosterone deve avere anche avuto un buon effetto sul presunto ipogonadismo del giovane uomo politico: nel 1963 questi confiderà al Primo Ministro britannico MacMillan di accusare terribili cefalee restando troppo a lungo senza una donna.

Nel 1946, Kennedy accusa intense "fitte dolorose urinarie", verosimilmente secondarie a un'uretrite cronica, forse blenorragica, risalente agli anni del College (egli era contrario al rapporto protetto né era tanto propenso alla procreazione controllata), al tempo ascritto a "prostatite cronica aspecifica", trattata con successo con sulfamidici. Si sa che, nel Maggio del 1955, era insorta una "marcata prostatite (accertata alla cistoscopia) con dolore durante la minzione e l'eiaculazione, da "infezione delle vie urinarie".

Il Presidente fumava molti sigari. Al termine della Campagna elettorale del 1960 appariva "esausto" e, alla conferenza stampa tenuta subito dopo l'elezione, "le sue mani tremavano"; in quell'occasione ha fatto anche cenno alle voci che correvano intorno alla propria salute: "Io non ho mai avuto il morbo di Addison, ha dichiarato in modo perentorio: ho condotto la mia campagna elettorale in piena salute e lo sono ancora".

Ma ancora settimane dopo non appariva "né sicuro, né lucido, ma sonnolento" e non mostrava ancora nessuna voglia di riprendere il lavoro.

\section{Tanti farmaci}

Una patologia così complessa non poteva non comportare una terapia altrettanto complessa e variegata, sia a causa delle incertezze al tempo esistenti circa la vera patogenesi del male sia per il vorticoso succedersi dell'avvento e dell'evoluzione dei farmaci (segnatamente dei corticosteroidi naturali e di sintesi.) Il che spiega, per esempio, le rapide varianti del peso corporeo e dell'aspetto stesso di John Kennedy, che appariva a volte emaciato e snello e, in altre occasioni, "appesantito" e con le guance gonfie. Per la stessa ragione, relativamente al colorito del viso, le testimonianze (specie in rapporto al periodo in cui sono state raccolte) risultano alquanto contrastanti: spesso il colorito "bronzino" (proprio dell'Addison) che caratterizzava il suo viso in ogni stagione 
veniva sottovalutato o ascritto ad "abbronzatura" solare. In pratica, per tutte queste ragioni e per la complessità del quadro clinico (non esclusa la depressione, che, peraltro, rientra nel morbo di Addison non trattato ma non in quello trattato (5)), negli ultimi otto anni Kennedy era arrivato a prendere 10-12 farmaci al giorno, tra antiflogistici, antidolorifici (specialmente novocaina), steroidi, miorilassanti, antianemici, antisettici urinari, ansiolitici e sonniferi.

Per esempio, $500 \mathrm{mg}$ di vitamina $\mathrm{C} / 2,10 \mathrm{mg} /$ die di idrocortisone, $2.5 \mathrm{mg}$ di prednisone $/ 2,10 \mathrm{mg}$ di metiltestosterone/die, $25 \mathrm{mcg}$ di liothyronina/2 e difenilossilato cloridrato+solfato di atropina, 2 compresse in base alle esigenze. Nonostante ciò, durante la crisi di Cuba del 1962 (durante la quale si sfiorò la III Guerra Mondiale) il Presidente non ha dato mai segni di non essere in grado di fronteggiare una situazione di tale gravità. In realtà, per l'intero periodo della Presidenza, JFK è stato trattato con testosterone e idrocortisone che, a dosaggio inappropriato, potrebbe essere stato causa dell'osteoporosi che certamente gli provocava dolori dorsali lancinanti, ai quali si univano quelli secondari alle lesioni vertebrali subite nell'Agosto del 1943 nel Sud-Est Pacifico in occasione dell'affondamento della motonave PT-109 su cui era imbarcato. Dolori che lo rendevano "in costanti gravi difficoltà", impedendogli persino di chinarsi per allacciarsi le scarpe e obbligandolo spesso a muoversi con le stampelle. Indossava, perciò, un busto dotato di stecche rigide di metallo, fissato al torace con delle fasce imbottite. Secondo gli esperti, sarebbe stato proprio questo tipo di busto a costargli la vita il giorno dell'attentato (Figg. 2, 3): infatti, se non l'avesse indossato, dopo essere stato colpito dal primo colpo, il suo corpo (quindi non così irrigidito dal busto) sarebbe scivolato nel sedile e non avrebbe consentito al killer di mirare con tanta precisione alla testa. Tragica fine per uno dei più famosi pazienti di Addison della storia.

\section{Riassunto}

Il morbo di Addison era stato diagnosticato a John Fitzgerald Kennedy, trentacinquesimo Presidente degli Stati Uniti, già agli inizi del 1940, ma era stato tenuto segreto per ragioni politiche e diplomatiche. Insorto probabilmente negli anni della guerra del Pacifico e trattato con atabrina, esso subì, tuttavia, un aggravamento nell'Ottobre del 1947, quando si temette addirittura l'esito letale.

Dall'esame autoptico eseguito nel 1963 in occasione del mortale attentato, risulterà trattarsi di una "sindrome poliendocrina autoimmune di tipo 2 (APS-2)", che può notoriamente coinvolgere più patologie come "morbo di Addison, ipotiroidismo e altre discrinie". L'Autoimmune Polyendocrine Syndrome (APS) varietà 2 viene oggi considerata malattia autoimmune contrassegnata, oltre che dalla presenza (costante) di morbo di Addison, anche da tireopatia autoimmune e/o diabete mellito tipo 1. La complessa (e, per l'epoca, ancora incerta) associazione di altri fattori patologici (compresa la depressione) comportò il simultaneo impiego di un congruo numero di farmaci: negli ultimi otto anni di vita Kennedy arrivò a prendere 10-12 farmaci al giorno tra antiflogistici, antidolorifici, steroidi, antianemici, antisettici urinari, miorilassanti, ansiolitici e sonniferi.

Parole chiave: J.F. Kennedy, Morbo di Addison, Autopsia, Poliendocrinopatia autoimmune di tipo 2

Dichiarazione di conflitto di interessi: L'Autore dichiara di non avere conflitto di interessi.

Indirizzo dell'Autore:

Dr. Luciano Sterpellone

Via Nicotera 29

00195 Roma

1.sterpellone@libero.it

\section{Bibliografia}

1. Addison T. 1855 on the constitutional and local effects of disease of the suprarenal capsules. In a collection of the published writing of the late Thomas Addison, M.D., physician to Guy's Hospital London: Sydenham Society 1868. Reprinted in Medical Classics 1937; 2: 244-93.

2. Neufeld M, Blizzard RM. Polyglandular autoimmune diseases. In: Symposium on autoimmune aspects of endocrine disorders.
Pinchera A, Doniach D, Fenzi GF, Baschieri L (Eds). Academic Press, New York 1980: 357-65.

3. Lee R Mandel. Endocrine and autoimmune aspects of the health history of John F. Kennedy. Annals od Internal Medicine 2009; 151: 350-4.

4. Michels WA, Gottlieb PA. Autoimmune polyglandular syndromes. Nature Reviews Endocrinology 2010; 6: 270-7.

5. Betterle $\mathrm{C}$, Zanchetta R. Update on autoimmune polyendocrine syndromes (APS). Acta Biomed 2003; 74; 9-33. 